\title{
On a Logical Foundation for Explicit Substitutions
}

\author{
Frank Pfenning \\ Department of Computer Science \\ Carnegie Mellon University \\ fp@cs.cmu.edu \\ http://www.cs.cmu.edu/ $f p$
}

Traditionally, calculi of explicit substitution [1] have been conceived as an implementation technique for $\beta$-reduction and studied with the tools of rewriting theory. This computational view has been extremely fruitful (see [2] for a recent survey) and raises the question if there may also be a more abstract underlying logical foundation.

Some forms of explicit substitution have been related to cut in the intuitionistic sequent calculus 3 . While making a connection to logic, the interpretation of explicit substitutions remains primarily computational since they do not have a reflection at the level of propositions, only at the level of proofs.

In recent joint work 4, we have shown how explicit substitutions naturally arise in the study of intuitionistic modal logic. Their logical meaning is embodied by a contextual modality which captures all assumptions a proof of a proposition may rely on. Explicit substitutions mediate between such contexts and therefore, intuitively, between worlds in a Kripke-style interpretation of modal logic.

In this talk we review this basic observation about the logical origin of explicit substitutions and generalize it to a multi-level modal logic. Returning to the computational meaning, we see that explicit substitutions are the key to a $\lambda$ calculus where variables, meta-variables, meta-meta-variables, etc. can be unified without the usual paradoxes such as lack of $\alpha$-conversion. We conclude with some speculation on potential applications of this calculus in logical frameworks or proof assistants.

\section{References}

1. Abadi, M., Cardelli, L., Curien, P.L., Lévy, J.J.: Explicit substitutions. Journal of Functional Programming 1(4), 375-416 (1991)

2. Kesner, D.: The theory of calculi with explicit substitutions revisited. Unpublished manuscript (October (2006)

3. Herbelin, H.: A lambda-calculus structure isomorphic to Gentzen-style sequent calculus structure. In: Pacholski, L., Tiuryn, J. (eds.) CSL 1994. LNCS, vol. 933, pp. 61-75. Springer, Heidelberg (1995)

4. Nanevski, A., Pfenning, F., Pientka, B.: Contextual modal type theory. Transactions on Computational Logic (To appear, 2007) 